\title{
Le froid en fromagerie ${ }^{(1)}$
}

\author{
par \\ Michel ANQUEZ \\ (I.N.A. 1938-1943) \\ Ingénieur en chef du Génie Rural, des Eaux et des Forêts \\ Directeur de l'Institut International du Froid
}

\section{INTRODUCTION}

On a pu considérer le fromage, aliment à base de lipides et de protides, comme " une véritable culture de micro-organismes ". Le rôle du froid en fromagerie consistera donc, non pas à arrêter toute fermentation, mais au contraire à orienter, grâce à une température et une humidité relative convenables, le développement des microorganismes favorables ou des actions enzymatiques souhaitables. C'est seulement en fin de maturation que le froid jouera son rôle classique d'agent de conservation.

En fromagerie, l'action du froid permet essentiellement de régulariser et d'améliorer la qualité des produits obtenus : il faudra agir avec souplesse et mesure, en maîtrisant, grâce au conditionnement d'air des locaux, la température et l'humidité relative, indépendamment des conditions extérieures. Mais le fromage, produit vivant, exige que les équipements frigorifiques tiennent soigneusement compte des besoins particuliers de la matière traitée, spécialement délicate.

Si l'on trouve des utilisations du froid presque à toutes les étapes de la fabrication des fromages, elles varieront d'un type de fromage à un autre. C'est pourquoi il convient de rappeler brièvement les conditions de traitement thermique des principaux types de fromages avant de situer les différentes applications du froid : avant fabrication, au cours de l'emprésurage, du hâlage, de l'affinage et de la conservation.

\section{Les conditions de traitement thermique des principaux types de fromage}

Le tableau suivant (tab. 1), tiré de l'ouvrage de MM. Jacquet et Thévenot, "le lait et le froid ", illustre bien la complexité du pro-

(1) Cahiers des Ingénieurs agronomes, 1974, nº 282. 
blème à résoudre ; il montre que, d'un type de fromage à un autre, les conditions de température et d'humidité relative, résultant de l'expérience et de l'observation professionnelles, varient considérablement, ce qui n'est d'ailleurs pas pour surprendre, quand on sait la diversité des produits finis.

\section{Le refroidissement du lait avant fabrication}

Traditionnellement, dans les régions de montagne où l'on fabrique les fromages à pâte cuite, la traite du soir était refroidie ; pour ce faire, le lait était mis dans des récipients peu profonds, appelés " rondots " ou « bagnolets ", placés dans une "chambre à lait ", sur des étagères parcourues par un courant d'eau fraîche. Aujourd'hui cette technique, utilisée depuis le $\mathrm{XVIII}^{\mathrm{e}}$ siècle, est de plus en plus remplacée par un refroidissement plus rapide sur un échangeur à ruissellement,

Pour les autres types de fromages, le refroidissement avant fabrication est également à conseiller, mais il ne doit pas être trop poussé : on a constaté, en effet, que si le lait est refroidi à la ferme, à des températures inférieures à $4^{\circ} \mathrm{C}$, la durée de coagulation par la présure peut augmenter considérablement (de 10 à 200 p. 100 selon des études irlandaises). La généralisation du refroidissement du lait à la production posera, dans les zones fromagères, un problème qui mérite dès maintenant des études approfondies.

\section{L'emprésurage à froid}

Si le rafraîchissement du lait avant fabrication est utilisé depuis très longtemps, la technique de l'emprésurage à froid, ou plus exactement en deux temps, est toute récente ; elle n'a d'ailleurs guère dépassé le stade expérimental. En 1942, l'Anglais Berridge a découvert que l'on pouvait distinguer deux phases dans l'action de la présure sur le lait : une action enzymatique et une action de coagulation. Dans la phase primaire, la présure se comporte comme une estérase, agissant sur la caseine $\%$; cette action, qui ne nécessite pas la présence de calcium, peut se dérouler entre 0 et $10^{\circ} \mathrm{C}$. La phase secondaire provoque la précipitation de la caséine sous forme de paracaséinate de chaux ; cette action de coagulation proprement dite ne peut se dérouler à une température inférieure à $20^{\circ} \mathrm{C}$ environ.

Berridge a établi que les deux actions de la présure pouvaient être dissociées, en utilisant les différences de températures auxquelles elles interviennent. A basse température, seule l'action enzymatique se produit, sans coagulation ; mais si on réchauffe ce lait emprésuré à froid, la coagulation se produit en quelques secondes.

A partir de 1960, trois types de procédés ont été proposés, qui reposent sur cette découverte ; tous sont liés aux tentatives de fabrication du fromage en continu. 
TABLEAU 1. - Conditions de traitement thermique de divers types de fromages

\begin{tabular}{|c|c|c|c|c|c|c|}
\hline & $\begin{array}{l}\text { Empré- } \\
\text { surage }\end{array}$ & $\begin{array}{l}\text { Egouttage } \\
\text { ou pressage }\end{array}$ & Salage & Hâlage & Affinage & $\begin{array}{l}\text { Conser- } \\
\text { vation }\end{array}$ \\
\hline Fromages frais (blancs) & 14 à $21^{\circ} \mathrm{C}$ & & & & & $\begin{array}{l}0 \mathrm{a}+5^{\circ} \mathrm{C} \\
(2 \text { à } 7 \mathrm{j})\end{array}$ \\
\hline $\begin{array}{l}\text { Fromages à pâte molle : } \\
\text { Camembert }\end{array}$ & 27 à $32^{\circ} \mathrm{C}$ & 18 à $30^{\circ} \mathrm{C}$ & $\begin{array}{l}12 \underset{\mathrm{à}}{ } 15^{\circ} \mathrm{C} \\
(1 \mathrm{j})\end{array}$ & $\begin{array}{c}12 \text { à } 14^{\circ} \mathrm{C} \\
\text { HR } 85 \text { à } 90 \% \\
\text { ventilation } \\
\text { assez forte } \\
(10 \mathrm{j})\end{array}$ & $\begin{array}{l}8 \text { à } 10^{\circ} \mathrm{C}-\mathrm{HR} 90 \% \\
\text { faible ventilation } \\
(1 \text { à } 2 \text { semaines })\end{array}$ & \\
\hline $\begin{array}{l}\text { Fromages à pâte ferme non cuite } \\
\text { Tilsitt (non pressé) }\end{array}$ & $\begin{array}{l}30 \text { à } 32^{\circ} \mathrm{C} \\
\text { puis } \\
\text { réchauffage } \\
\text { à } 38-15^{\circ} \mathrm{C}\end{array}$ & 18 à $20^{\circ} \mathrm{C}$ & $\begin{array}{l}12 \text { à } 15^{\circ} \mathrm{C} \\
\text { HR } 95 \% \\
(2 \text { à } 3 \mathrm{j})\end{array}$ & & $\begin{array}{l}12 \text { à } 16^{\circ} \mathrm{C}-\mathrm{HR} 95 \% \\
(5 \text { mois environ) }\end{array}$ & $\begin{array}{l}+2^{\circ} \mathrm{C} \\
\text { HR } 90 \%\end{array}$ \\
\hline Bel Paese (non pressé) & 37 à $40^{\circ} \mathrm{C}$ & $\begin{array}{l}27 \text { à } 28^{\circ} \mathrm{C} \\
(5 \text { à } 6 \mathrm{~h})\end{array}$ & $\begin{array}{l}12 \text { à } 15^{\circ} \mathrm{C} \\
\text { en saumure } \\
(10 \text { à } 12 \mathrm{~h})\end{array}$ & & $\begin{array}{l}+4^{\circ} \mathrm{C}-\mathrm{HR} 90 \% \\
\quad(30 \text { à } 45 \mathrm{j})\end{array}$ & \\
\hline $\begin{array}{l}\text { Hollande, Edam ou Saint-Paulin } \\
\text { (pressés) }\end{array}$ & 30 à $35^{\circ} \mathrm{C}$ & $18^{\circ} \mathrm{C}$ & $\begin{array}{l}12 \text { à } 15^{\circ} \mathrm{C} \\
(3 \text { à } 5 \mathrm{j})\end{array}$ & & $\begin{array}{c}12 \text { à } 15^{\circ} \mathrm{C}-\mathrm{HR} 90 \% \\
\text { faible ventilation } \\
\text { (3 semaines à } 1 \text { mois) }\end{array}$ & $\begin{array}{c}0 \mathrm{a}+5^{\circ} \mathrm{C} \\
\text { HR } 90 \% \\
\text { faible ventil. }\end{array}$ \\
\hline Cheddar (pressé) & $\begin{array}{l}20 \text { à } 30^{\circ} \mathrm{C} \\
\text { puis } \\
\text { réchauffage } \\
\text { à } 35-10^{\circ} \mathrm{C}\end{array}$ & $\begin{array}{l}20^{\circ} \mathrm{C} \\
(1 \text { à } 3 \mathrm{j})\end{array}$ & $20^{\circ} \mathrm{C}$ & & $\begin{array}{l}6 \text { à } 10^{\circ} \mathrm{C}-\mathrm{HR} 85 \% \\
(2 \text { à } 5 \text { mois })\end{array}$ & $\begin{array}{l}0 \text { à }+2^{\circ} \mathrm{C} \\
\text { HR } 70 \\
\text { à } 75 \%\end{array}$ \\
\hline
\end{tabular}




\begin{tabular}{|c|c|c|c|c|c|c|}
\hline Cheshire (pressé) & $\begin{array}{l}30^{\circ} \mathrm{C} \\
\text { puis réchauf. } \\
\text { à } 32-33^{\circ} \mathrm{C}\end{array}$ & $\begin{array}{l}21^{\circ} \mathrm{C} \\
(2 \mathrm{j})\end{array}$ & $21^{\circ} \mathrm{C}$ & & $\begin{array}{c}13 \text { à } 16^{\circ} \mathrm{C} \\
(1 \text { à } 3 \text { mois })\end{array}$ & \\
\hline $\begin{array}{l}\text { Fromages à pâte cuite : } \\
\text { Emmental }\end{array}$ & 30 à $35^{\circ} \mathrm{C}$ & 18 à $20^{\circ} \mathrm{C}$ & $\begin{array}{l}10 \text { à } 15^{\circ} \mathrm{C} \\
\text { en saumure } \\
(2 \text { à } 3 \mathrm{j})\end{array}$ & & $\begin{array}{l}1^{\circ} \text { Cave froide : } \\
10 \text { à } 15^{\circ} \mathrm{C}-\text { HR } 85 \% \\
(10 \mathrm{j} \text { environ }) \\
2^{\circ} \text { Cave chaude : } \\
16 \text { à } 25^{\circ} \mathrm{C}-\text { HR } 85 \%\end{array}$ & $\begin{array}{l}10 \text { à } 12^{\circ} \mathrm{C} \\
\text { HR } 80 \% \\
\text { (plusieurs } \\
\text { mois) }\end{array}$ \\
\hline Gruyère-Comté & 30 à $35^{\circ} \mathrm{C}$ & 18 à $20^{\circ} \mathrm{C}$ & $\begin{array}{l}10 \text { à } 15^{\circ} \mathrm{C} \\
\text { en saumure } \\
(2 \text { à } 3 \mathrm{j})\end{array}$ & & $\begin{array}{c}1^{\circ} \text { Cave froide : } \\
10 \text { à } 15^{\circ} \mathrm{C}-\mathrm{HR} 85 \% \\
(10 \mathrm{j}) \\
2^{\circ} \text { Cave chaude : } \\
16 \text { à } 18^{\circ} \mathrm{C} \\
(30 \text { à } 40 \mathrm{j})\end{array}$ & $\begin{array}{c}10 \text { à } 12^{\circ} \mathrm{C} \\
\text { HR } 80 \\
\text { à } 85 \% \\
\text { (plusieurs } \\
\text { mois) }\end{array}$ \\
\hline Parmesan & $\begin{array}{l}35^{\circ} \mathrm{C} \\
\text { puis } \\
\text { réchauffage } \\
\text { à } 55-58^{\circ} \mathrm{C}\end{array}$ & $\begin{array}{c}15 \text { à } 16^{\circ} \mathrm{C} \\
(20 \mathrm{~h})\end{array}$ & $\begin{array}{c}15 \text { à } 16^{\circ} \mathrm{C} \\
(30 \mathrm{j})\end{array}$ & & $\begin{array}{c}16 \text { à } 18^{\circ} \mathrm{C}-\text { HR } 80 \% \\
\text { Ventilation (environ } 1 \text { an) }\end{array}$ & $\begin{array}{l}\text { Plusieur } \\
\text { années }\end{array}$ \\
\hline Fromages à pâte persillée (bleus) & & & & & & \\
\hline Roquefort, Bleus d'Auvergne & 28 à $30^{\circ} \mathrm{C}$ & $\begin{array}{l}18^{\circ} \mathrm{C} \\
\text { HR } 90 \% \\
(3 \text { à } 5 \mathrm{j}) \\
\text { ensemen- } \\
\text { cement }\end{array}$ & $\begin{array}{l}8 \text { à } 10^{\circ} \mathrm{C} \\
\text { HR } 95 \\
\text { à } 97 \%\end{array}$ & & $\begin{array}{c}4 \text { à } 10^{\circ} \mathrm{C}-\mathrm{HR} 95 \text { à } 100 \% \\
\text { Ventilation assez forte }\end{array}$ & $\begin{array}{c}0^{\circ} \mathrm{C} \\
\mathrm{HR} 75 \\
\text { à } 80 \% \\
\text { Ventilation } \\
\text { modérée }\end{array}$ \\
\hline Gorgonzola & 32 à $33^{\circ} \mathrm{C}$ & $\begin{array}{l}16 \text { à } 20^{\circ} \mathrm{C} \\
(3 \text { à } 4 \mathrm{j})\end{array}$ & $\begin{array}{l}16 \text { à } 20^{\circ} \mathrm{C} \\
(20 \text { à } 21 \mathrm{j})\end{array}$ & $\begin{array}{l}13 \text { à } 16^{\circ} \mathrm{C} \\
\text { HR } 75 \\
\text { à } 80 \% \\
(20 \text { à } 30 \mathrm{j})\end{array}$ & $\begin{array}{c}\text { Premier stade : } \\
9 \text { à } 11^{\circ} \mathrm{C}-\text { HR } 85 \text { à } 90 \% \\
(1 \text { mois environ }) \\
\text { Deuxième stade : } \\
1 \text { à } 7^{\circ} \mathrm{C}-\text { HR } 90 \% \\
(2 \text { mois environ })\end{array}$ & $\begin{array}{c}4 \text { à } 5^{\circ} \mathrm{C} \\
\text { HR } 90 \%\end{array}$ \\
\hline Fromages fondus & & & & & & 14 à $15^{\circ} \mathrm{C}$ \\
\hline
\end{tabular}


Berridge lui-même a suggéré une technique qui consiste à emprésurer le lait à $4^{\circ} \mathrm{C}$, et à le maintenir à cette température pendant plusieurs heures ; la coagulation se produit à $50-70^{\circ} \mathrm{C}$ : elle est presque immédiate.

Les Hollandais de l'Institut Néerlandais de Recherches Laitières (NIZO) ont mis au point un procédé et une machine (procédé Nicoma) de fabrication de fromage en continu, dont la première phase d'emprésurage possède les caractéristiques suivantes : emprésurage à $2^{\circ} \mathrm{C}$ (actuellement, les Hollandais estiment qu'un emprésurage à $6^{\circ} \mathrm{C}$ donne les mêmes résultats); stockage : 2 à $3 \mathrm{~h}$; réchauffage à $30^{\circ} \mathrm{C}$ permettant une coagulation en $100 \mathrm{~s}$.

Le procédé Stenne-Hutin ( $\mathrm{SH} 13$ ) diffère des deux autres par le fait que l'on part de lait concentré (3/1), standardisé et pasteurisé. L'emprésurage à froid est réalisé à $10^{\circ} \mathrm{C}$, pendant 30 à $40 \mathrm{mn}$; la coagulation est instantanée par injection d'eau chaude.

\section{Egouttage, pressage, salage}

Pour certains types de fromages, les locaux d'égouttage, de pressage et de salage sont à refroidir en été à des températures comprises entre 12 et $18^{\circ} \mathrm{C}$. On utilise très généralement la circulation d'air forcée. Le niveau de l'humidité relative importe peu, car la durée du séjour des fromages dans ces locaux est faible : en outre, le fromage perd de l'eau à ce stade de fabication, si bien qu'une humidité relative plutôt faible $(80-85$ p. 100$)$, plus facile à réaliser, est ici plutôt un avantage.

Lorsque le salage se fait par voie humide, dans une saumure à $10-12^{\circ} \mathrm{C}$, un refroidissement de la saumure est à préconiser, au moins en été, si l'usine est approvisionnée en eau à température élevée.

\section{Hâlage}

Ce stade de fabrication, particulier aux pâtes molles, consiste à enlever lentement l'eau en excès dans le fromage; on cherche donc un effet de séchage contrôlé. Ce séchage est obtenu en faisant circuler dans le hâloir un air, dont l'humidité relative est maintenue à $85-90$ p. 100 , à une température de 12 à $14^{\circ} \mathrm{C}$. L'air peut se charger de la vapeur d'eau dégagée par le fromage et cette vapeur d'eau se condense sur l'évaporateur de l'installation frigorifique : dans un local de hâlage, le rôle essentiel de l'évaporateur est de piéger l'eau issue des fromages, pour que le phénomène de déshydratation ménagée puisse se poursuivre.

Il en résulte trois conséquences :

- la maîtrise de la température et de l'humidité relative étant facilement assurée par l'installation frigorifique, les locaux de hâlage ne doivent pas comporter de fenêtres ; 
- comme on cherche à créer des besoins de froid pour faire fonctionner l'évaporateur, il est recommandé de ne pas isoler trop fortement les parois ;

- lorsque la température extérieure est inférieure à la température requise pour le hâlage $\left(12-14^{\circ} \mathrm{C}\right)$, il est nécessaire de créer artificiellement des besoins de froid, donc de chauffer le local, pour que l'évaporateur puisse fonctionner et jouer son rôle de piège à eau. Ce chauffage peut être obtenu par des radiateurs (à eau chaude ou électriques) placés dans le hâloir, ou, ce qui est préférable, en insérant une batterie de chauffage, en aval de la batterie froide, dans le conditionneur d'air qui dessert le local.

En outre, compte tenu des caractéristiques demandées au local, il est souhaitable de prévoir une circulation d'air assez active, et d'employer des systèmes de répartition d'air sans gaines (pour éviter le développement de moisissures indésirables).

Enfin, comme la matière première n'est pas constante, il serait préférable d'avoir un local de hâlage par jour de fabrication (donc $n$ hâloirs si le hâlage dure $n$ jours ; dans la pratique $n$ est de l'ordre de 8 à 10). En fait, sauf pour les très grandes fromageries, on est amené à un compromis, et on s'en tient à des hâloirs de $50 \mathrm{~m}^{2}$ environ. On a quelquefois cherché à refroidir de façon indépendante des travées d'un grand hâloir, afin de pouvoir faire varier, dans chaque travée, température et humidité relative ; les résultats ont souvent été décevants.

\section{Affinage}

Cette étape de la fabrication, essentielle, intéresse toutes les catégories de fromages (à l'exception des pâtes fraîches). C'est aussi celle où l'intervention du froid est délicate, non pas à cause des températures à obtenir, mais par suite de l'humidité relative très élevée que l'on doit maintenir (95 p. 100 et plus), ce qui est difficile à réaliser.

L'importance de la circulation d'air résultera ici d'un compromis : l'homogénéité de l'atmosphère, aussi bien pour la température que pour l'humidité relative, demande une ventilation d'air active, qui peut par contre, entraîner des accidents de fabrication (la " gerce " du gruyère), si le courant d'air est trop rapide et pas assez humide.

La solution de ces problèmes entraîne un certain nombre de conséquences quant à l'équipement frigorifique.

Tout d'abord, en ce qui concerne la construction des locaux, les murs seront aveugles, afin de limiter au maximum les échanges de chaleur avec l'extérieur. Dans le même ordre d'idées, on renforcera l'isolation; la ventilation pourra être en effet réduite ainsi qu'il est souhaitable, parce que la chaleur à évacuer, qui sera plus faible 
si l'isolation est plus forte, est liée au débit d'air en circulation. En outre, une isolation renforcée évitera, pendant l'hiver, la condensation d'eau sur la face intérieure des parois (ce qui entraîne une dessiccation des fromages) : ceci est évidemment surtout valable dans les climats froids des montagnes.

Le refroidissement pourra se faire par détente directe d'un fluide frigorigène, par eau glacée (si la température d'affinage du fromage est supérieure à 7-8 $\mathrm{C}$ ), éventuellement par saumure (mais qui entraîne des risques sérieux de corrosion).

La distribution d'air peut être de type naturel ou forcé. La convection naturelle, intéressante parce qu'elle permet plus aisément d'obtenir des humidités relatives très élevées, entraîne malheureusement un manque d'homogénéité de l'atmosphère du local ; on ne l'emploiera que dans des chambres petites (moins de $100 \mathrm{~m}^{3}$ ), en utilisant des appareils de distribution du froid plafonniers (audessus des allées de préférence), muraux ou les deux à la fois. On pourrait songer à utiliser des chambres à double paroi, méthode grâce à laquelle on peut créer dans un local une humidité relative très élevée, la ventilation n'intervenant que dans la double paroi : mais cette méthode n'est pas employée à notre connaissance sans doute à cause de son prix élevé.

La convection forcée est en fait le système le plus fréquemment adopté. On peut maintenir des humidités relatives très élevées, grâce à un certain nombre de précautions constructives : forte isolation des parois, faible écart de température entre l'air de la chambre et les surfaces froides, faible différence de température de l'agent de refroidissement à l'entrée et à la sortie de la batterie froide : ces précautions entraînent évidemment une augmentation des dépenses d'investissement.

On peut aussi songer à utiliser des frigorifères humides à eau glacée pour obtenir une humidité relative très élevée. L'air de la chambre est envoyé dans une cabine où il se refroidit et s'humidifie au contact d'eau glacée finement pulvérisée ; l'air sort saturé de cette cabine de conditionnement d'air spéciale (quelquefois appelée "laveur d'air»).

Si l'équipement des locaux d'affinage est mal conçu, et en particulier si l'air de circulation est trop sec, on peut avoir recours à quelques palliatifs, dont l'action apportera un remède partiel aux imperfections constatées : par exemple l'arrosage du sol, la pulvérisation d'eau dans le local, l'injection de vapeur d'eau (dans les gaines ou aux bouches de soufflage d'air froid).

\section{Conservation}

On retrouve ici l'utilisation normale du froid qui consiste à limiter et même si possible annuler l'évolution des produits. 
En régime de réfrigération, ce qui est le cas normal, on cherche surtout à éviter la déshydratation, à craindre lorsque les fromages sont nus : dans ce cas, on refroidit en convection naturelle, ou en ventilation avec un très faible coefficient de brassage (de l'ordre de 10). On maintient généralement des températures comprises entre $0^{\circ} \mathrm{C}$ et $+2^{\circ} \mathrm{C}$.

Plus rarement, on congèle des fromages, encore que des expériences récentes semblent donner d'excellents résultats, notamment sur les fromages blancs, et également sur les pâtes molles. De même, on congèle assez souvent des gruyères, avant affinage, afin d'arrêter complètement l'évolution (si par exemple le marché est mauvais) ; la fabrication reprendra normalement après réchauffage.

\section{Quelques applications particulières du froid}

Outre cette utilisation généralisée en fromagerie classique, on rencontre quelques applications particulières du froid.

La fabrication du yaourt en est un exemple (si l'on considère le yaourt comme un fromage). Après étuvage, intervient un refroidissement rapide à $6-7^{\circ} \mathrm{C}$, soit dans un fort courant d'air (tunnel de réfrigération rapide), soit dans un courant d'eau glacée dans lequel baignent les pots. Ces opérations se font de plus en plus en continu. On a aussi procédé à des essais de lyophilisation.

Autre cas : la fabrication du type " cottage cheese " aux EtatsUnis. Le caillage se fait à $38^{\circ} \mathrm{C}$ dans de grands bacs (4 à $5 \mathrm{t}$ ) ; le caillé est découpé, puis porté à $48-50^{\circ} \mathrm{C}$ pendant $30 \mathrm{mn}$, et égoutté ; enfin, a lieu le lavage dans les mêmes bacs, une première fois avec de l'eau à température ambiante, puis, après égouttage intermédiaire, une seconde fois avec de l'eau glacée à $24^{\circ} \mathrm{C}$. Un égouttage final termine la fabrication.

L'application au fromage de chèvre de la congélation et de la conservation à basse température se développe (la quantité de fromage traité par le froid atteint, en période de lactation maximum, plus de $2 \mathrm{t} / \mathrm{j}$ dans les usines les plus importantes). La conservation est la meilleure lorsque le fromage est congelé avant affinage, au stade de caillé égoutté frais. La congélation est faite rapidement en tunnel, à une température de $-35^{\circ} \mathrm{C}$ à $-40^{\circ} \mathrm{C}$ et le caillé est stocké en chambre froide, à une température au plus égale à $-20^{\circ} \mathrm{C}$ pendant 4 à 5 mois (on a constaté quelques défauts dus à l'oxydation de la matière grasse, après 5 mois de stockage à $-20^{\circ} \mathrm{C}$ ). Il faut noter aussi que le hâlage des fromages de chèvre se fait plus vite que celui des fromages de vache et que la quantité d'eau à enlever est plus importante : d'où la nécessité d'une installation frigorifique plus puissante d'une ventilation active ; il est encore plus nécessaire de chauffer. 
Enfin, les ferments utilisés en fromageries subissent aussi l'action du froid, soit par congélation, soit, de plus en plus, par lyophilisation.

\section{Conclusions}

La fromagerie : un secteur privilégié pour les applications du froid dans le domaine agricole et alimentaire. On y rencontre, à un moment ou un autre de la fabrication, presque toutes les utilisations du froid, à différents niveaux de température : lyophilisation, congélation, réfrigération, conditionnement d'air. On a essayé presque tous les modes de transmission du froid, qu'il s'agisse des agents de refroidissement (détente directe, saumure, eau glacée) ou des systèmes de distribution d'air (convection naturelle, convection forcée).

On trouve même des utilisations originales destinées à apporter une solution au problème délicat de l'obtention d'humidités relatives très élevées, notamment en employant les frigorifères humides. En outre, les applications du froid doivent toujours être conduites avec souplesse et mesure, pour maîtriser constamment l'évolution des processus enzymatiques et micro-organiques, responsables de la fabrication du fromage. C'est dire que la fromagerie est un domaine choisi pour le frigoriste.

Ce domaine est spécialement intéressant pour le frigoriste français, puisque d'une part la gamme des fromages de nos terroirs est variée à l'infini et que par conséquent la diversité des conditions d'application du froid est extrêmement grande, d'autre part parce que les quantité de fromages fabriqués en France dépassent 60000 t, et que de ce fait, le marché est largement ouvert. Ce n'est d'ailleurs pas un hasard si, pour ne citer que quelques exemples, la France est à la pointe du progrès pour les recherches entreprises sur la fabrication du fromage de chèvre et que le volume des locaux utilisant le froid pour le hâlage et l'affinage des fromages atteint actuellement $650000 \mathrm{~m}^{3}$ : nous avons, dans ce domaine, la deuxième place au monde, derrière les Etats-Unis, mais il est bien évident que les techniques utilisées sont beaucoup plus variées qu'aux Etats-Unis, où l'on fabrique essentiellement du cheddar.

Ainsi donc les applications du froid en fromagerie ont permis et permettront encore aux frigoristes français de mettre au point des méthodes qui, garantissant aux produits une plus grande régularité, maintiendront la prééminence de notre pays dans ce domaine original de notre production agricole.

\section{Bibliographie}

Jacquet (J.) et Thévenot (R.) (1961). - Le lait et le froid, 461 pages. Editions J. B. Baillière. 
THÉvenot (R.) (1964). - Cours d'installations pour le traitement frigorifique des denrées périssables. Ecole Nationale du Génie Rural. Editions Scientifiques Ribert.

ANQuez (M.) (1970). - Cours du froid à l'Institut d'Etudes Supérieures d'Industries et d'Economie Laitières (non publié).

Tiersonnier (B.) (1969). - Le froid en fromagerie de chèvre. Génie Rural, juin, 363-365.

Portmann (A.) (1970). - La congélation et le stockage du fromage de chèvre. Le Lait, mars-avril, 161-164.

A.P.R.I.A. (1971). - Les nouveaux procédés mécanisés et continus dans l'industrie alimentaire. Tome 5, Fromagerie. 